\title{
Phase Estimation of Coherent States with a Noiseless Linear Amplifier
}

\author{
Syed M Assad, ${ }^{1,}$ | Mark Bradshaw, ${ }^{1}$ and Ping Koy Lam ${ }^{1}$ \\ ${ }^{1}$ Centre for Quantum Computation and Communication Technology, \\ Research School of Physics and Engineering, The Australian National University, Canberra ACT 2601, Australia
}

\begin{abstract}
Amplification of quantum states is inevitably accompanied with the introduction of noise at the output. For protocols that are probabilistic with heralded success, noiseless linear amplification in theory may still possible. When the protocol is successful, it can lead to an output that is a noiselessly amplified copy of the input. When the protocol is unsuccessful, the output state is degraded and is usually discarded. Probabilistic protocols may improve the performance of some quantum information protocols, but not for metrology if the whole statistics is taken into consideration. We calculate the precision limits on estimating the phase of coherent states using a noiseless linear amplifier by computing its quantum Fisher information and we show that on average, the noiseless linear amplifier does not improve the phase estimate. We also discuss the case where abstention from measurement can reduce the cost for estimation.
\end{abstract}

\section{INTRODUCTION}

Quantum metrology is concerned with the measuring of a weak signal with the best achievable precision by using a quantum probe. One important example is in the detection of gravitational waves by measuring the phase difference of light. It would be beneficial if we could somehow amplify the signal prior to measurement. If the signal is encoded as the amplitude $\alpha$ of a coherent state $|\alpha\rangle$, a noiseless linear amplifier (NLA) can do just that. An NLA with an amplification gain $g>1$ transforms the coherent state $|\alpha\rangle$ to $|g \alpha\rangle$ 1], thereby amplifying the signal but not the noise. If this transformation can be performed deterministically, we would obtain a more precise estimate of the signal. Unfortunately, it is not possible to noiselessly amplify a quantum state [2]. But an approximate version of the NLA which works probabilistically is possible and has been realised by several experimental groups 3 - 7].

We investigate the precision of phase estimation of coherent states using a probabilistic NLA as shown schematically in Fig. 1. When the NLA successfully amplifies a coherent state, we are able to estimate the phase more precisely. However, when the amplification fails, we obtain a worse estimate of the phase than if we had not used the NLA. We show that on average, postselecting the successfully amplified events or using both successful and unsuccessful events of the NLA does not improve the precision of phase estimation. This is consistent with known results that by post-selecting based on the measurement outcomes, probabilistic metrology can result in improved quantum state estimation of the post-selected sub-ensemble [8] 10, but on average postselection cannot increase information [11,14. However with a different figure of merit, post-selection can help. This is the case for state discrimination when a cost is assigned to wrong guesses and for abstaining [15. For our case, by assigning a cost to rejecting a state and a cost

\footnotetext{
* E-mail: cqtsma@gmail.com
}

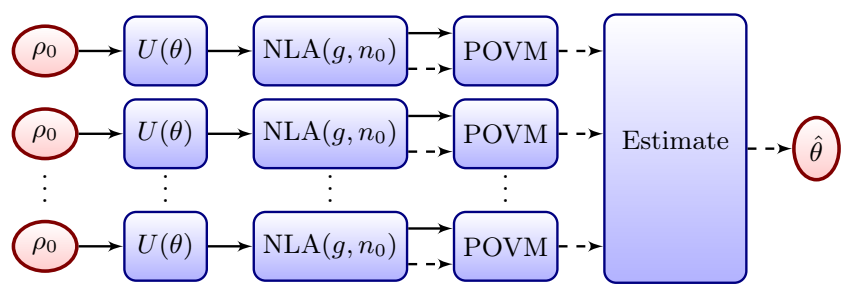

FIG. 1. Schematic of parameter estimation with an NLA. Identical probes in some initial state $\rho_{0}$ undergoes an interaction $U(\theta)$. The probes are then individually amplified in an NLA with gain $g$, and maximum photon number $n_{0}$. The NLA outputs a projected quantum state (solid arrow) and a classical variable (dashed arrow) indicating successful or failed amplification. Based on the classical variable, an optimal POVM is chosen to measure the quantum state. The output of these measurements are used to obtain an estimate for $\theta$.

for performing an estimator measurement, then by postselecting the successful outcome of the NLA and only performing the estimator measurement on these, we can achieve a desired precision at a lower cost.

\section{PHASE ESTIMATION}

To quantify the precision of an estimate, we shall use the quantum Fisher information [16-19]. Given a sample of $m$ identical and independent states $\rho_{\theta}$ that depend on some unknown parameter $\theta$ that we wish to estimate, the quantum Cramér Rao (QCR) bound states that the variance of an unbiased estimator $\hat{\theta}$ is bounded by

$$
\operatorname{Var}(\hat{\theta}) \geq \frac{1}{m J\left(\rho_{\theta}\right)},
$$

where $J\left(\rho_{\theta}\right)$ is the quantum Fisher information

$$
J\left(\rho_{\theta}\right)=\operatorname{Tr}\left[\rho_{\theta} \mathcal{L}^{2}\right] .
$$


The symmetric logarithmic derivative $\mathcal{L}$ is some Hermitian operator defined implicitly through

$$
\dot{\rho}_{\theta}=\frac{1}{2}\left(\rho_{\theta} \mathcal{L}+\mathcal{L} \rho_{\theta}\right)
$$

where an overdot is used to indicate a derivative with respect to $\theta$. The QCR bound is asymptotically attainable when $m \gg 1$ [20. A large Fisher information allows for a more precise estimate of the parameter $\theta$. Equivalently, a larger Fisher information allows a parameter $\theta$ to be estimated to the same precision from a smaller sample. For a pure state, $\rho_{\theta}=\left|\psi_{\theta}\right\rangle\left\langle\psi_{\theta}\right|$, we have $\dot{\rho}_{\theta}=\rho_{\theta} \dot{\rho}_{\theta}+\dot{\rho}_{\theta} \rho_{\theta}$ which indicates that we can take $\mathcal{L}=2 \dot{\rho}_{\theta}$. This gives $J\left(\rho_{\theta}\right)=4 \operatorname{Tr}\left[\rho_{\theta} \dot{\rho}_{\theta}^{2}\right]=4\left(\left\langle\dot{\psi}_{\theta} \mid \dot{\psi}_{\theta}\right\rangle+\left\langle\psi_{\theta} \mid \dot{\psi}_{\theta}\right\rangle^{2}\right)[21$.

We apply the above formalism to an NLA. The NLA we consider is implemented through a two outcome measurement device characterised by a gain $g>1$ and maximum amplified photon $n_{0} \in \mathbb{Z}^{+}$[22, 23. $n_{0}$ determines how closely the successfully amplified output from this device resembles the output from ideal NLA. A larger $n_{0}$ gives a more faithful approximation at the expense of a lower probability of success. The first measurement outcome corresponds to the operator

$$
E_{\mathrm{s}}=\sum_{n=0}^{n_{0}} g^{n-n_{0}}|n\rangle\left\langle n\left|+\sum_{n=n_{0}+1}^{\infty}\right| n\right\rangle\langle n|
$$

which heralds a successful amplification event and projects the input state $\rho_{\theta}$ to the state $\rho_{\mathrm{s}, \theta}=$ $E_{\mathrm{s}} \rho_{\theta} E_{\mathrm{s}} / \operatorname{Tr}\left[\rho_{\theta} E_{\mathrm{s}}^{2}\right]$. The successful amplification event occurs with probability $p_{\mathrm{s}}=\operatorname{Tr}\left[\rho_{\theta} E_{\mathrm{s}}^{2}\right]$. The second measurement outcome $E_{\mathrm{f}}=\sqrt{1-E_{\mathrm{s}}^{2}}$ corresponds to a failed amplification event which projects the input state to $\rho_{\mathrm{f}, \theta}=E_{\mathrm{f}} \rho_{\theta} E_{\mathrm{f}} / \operatorname{Tr}\left[\rho_{\theta} E_{\mathrm{f}}^{2}\right]$ and occurs with probability $p_{\mathrm{f}}=\operatorname{Tr}\left[\rho_{\theta} E_{\mathrm{f}}^{2}\right]$. We assume that $p_{\mathrm{s}}$ and $p_{\mathrm{f}}$ do not depend on $\theta$ which is true for the state that we shall consider later. From the states $\rho_{\mathrm{s}, \theta}$ we can construct $\hat{\theta}_{\mathrm{s}}$, an estimator of $\theta$, while from the states $\rho_{\mathrm{f}, \theta}$, we construct a second estimator $\hat{\theta}_{\mathrm{f}}$. Combining these two independent estimators, we arrive at a third estimator given by $\hat{\theta}_{\mathrm{NLA}}=\beta \hat{\theta}_{\mathrm{s}}+(1-\beta) \hat{\theta}_{\mathrm{f}}$. The weight

$$
\beta=\frac{V_{\mathrm{f}}}{V_{\mathrm{s}}+V_{\mathrm{f}}},
$$

where $V_{\mathrm{s}}$ and $V_{\mathrm{f}}$ denote the variances of $\hat{\theta}_{\mathrm{s}}$ and $\hat{\theta}_{\mathrm{f}}$, is chosen to minimise the variance of $\hat{\theta}_{\mathrm{NLA}}$. The variances $V_{\mathrm{s}}$ and $V_{\mathrm{f}}$ depend on the number of successful and failed amplification events denoted by $n_{\mathrm{s}}$ and $n_{\mathrm{f}}$ respectively. Hence the weight $\beta$ is also a function of number successfully amplified event $n_{\mathrm{s}}$. The variance of the estimator $\hat{\theta}_{\mathrm{NLA}}$ given $n_{\mathrm{s}}$ is

$$
\operatorname{Var}\left(\hat{\theta}_{\mathrm{NLA}} \mid n_{\mathrm{s}}\right)=\frac{1}{\frac{1}{V_{\mathrm{s}}}+\frac{1}{V_{\mathrm{f}}}} \geq \frac{1}{\left(n_{\mathrm{s}} J_{\mathrm{s}}+n_{\mathrm{f}} J_{\mathrm{f}}\right)},
$$

using the notation $J_{\mathrm{s}}=J\left(\rho_{\mathrm{s}, \theta}\right)$ and $J_{\mathrm{f}}=J\left(\rho_{\mathrm{f}, \theta}\right)$. We define $J_{\mathrm{NLA}}=\left(n_{\mathrm{s}} J_{\mathrm{s}}+n_{\mathrm{f}} J_{\mathrm{f}}\right) / m$, where $m=n_{\mathrm{s}}+n_{\mathrm{f}}$ is

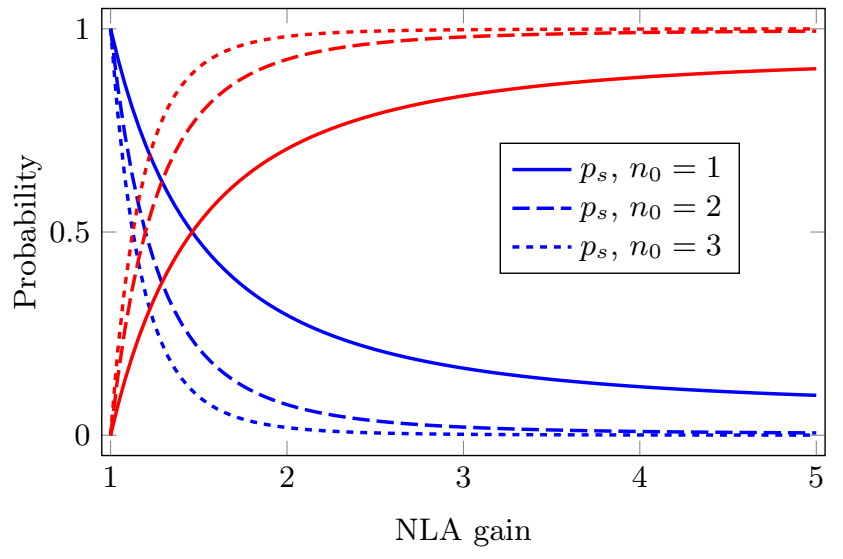

FIG. 2. Probability of successful (blue, decreasing) and failed (red, increasing) NLA amplification versus NLA gain. The blue and red lines add up to 1 . Input state has amplitude $r=0.25$.

the sample size. For a fixed $m, n_{\mathrm{s}}$ follows a binomial distribution with $\operatorname{Pr}\left(n_{\mathrm{s}}\right)=\left(\begin{array}{c}m \\ n_{\mathrm{s}}\end{array}\right) p_{\mathrm{s}}^{n_{\mathrm{s}}} p_{\mathrm{f}}^{n_{\mathrm{f}}}$. The unconditional variance of $\hat{\theta}_{\mathrm{NLA}}$ is then

$$
\begin{aligned}
\operatorname{Var}\left(\hat{\theta}_{\mathrm{NLA}}\right) & =\mathbb{E}_{n_{\mathrm{s}}}\left[\operatorname{Var}\left(\hat{\theta}_{\mathrm{NLA}} \mid n_{\mathrm{s}}\right)\right]+\operatorname{Var}\left(\mathbb{E}\left[\hat{\theta}_{\mathrm{NLA}} \mid n_{\mathrm{s}}\right]\right) \\
& =\mathbb{E}_{n_{\mathrm{s}}}\left[\operatorname{Var}\left(\hat{\theta}_{\mathrm{NLA}} \mid n_{\mathrm{s}}\right)\right],
\end{aligned}
$$

since $n_{\mathrm{s}}$ does not depend on $\theta$. For large $m, n_{\mathrm{s}} / m \rightarrow p_{\mathrm{s}}$ and $n_{\mathrm{f}} / m \rightarrow p_{\mathrm{f}}$ so that $J_{\mathrm{NLA}} \rightarrow p_{\mathrm{s}} J_{\mathrm{s}}+p_{\mathrm{f}} J_{\mathrm{f}}[14$.

We consider a coherent input state $\rho_{\alpha}=|\alpha\rangle\langle\alpha|$ with $\alpha=r e^{i \theta}$, where the amplitude $r$ is known and whose phase $\theta$ we wish to estimate. The quantum Fisher information for $\rho_{\alpha}$ is $J_{\alpha}=4 r^{2}$ [24]26]. Applying the NLA on the state $|\alpha\rangle$, we get one of the two outputs

$$
\begin{aligned}
\left|\psi_{\mathrm{s}}\right\rangle & =E_{\mathrm{s}}|\alpha\rangle \\
& =\frac{\exp \left(\frac{-r^{2}}{2}\right)}{\sqrt{p_{\mathrm{s}}(r)}}\left(\sum_{n=0}^{n_{0}}|n\rangle \frac{(g \alpha)^{n}}{\sqrt{n !} g^{n_{0}}}+\sum_{n=n_{0}+1}^{\infty}|n\rangle \frac{\alpha^{n}}{\sqrt{n !}}\right)
\end{aligned}
$$

or

$$
\begin{aligned}
\left|\psi_{\mathrm{f}}\right\rangle & =E_{\mathrm{f}}|\alpha\rangle \\
& =\frac{\exp \left(\frac{-r^{2}}{2}\right)}{\sqrt{p_{\mathrm{f}}(r)}} \sum_{n=0}^{n_{0}}|n\rangle \sqrt{1-\frac{g^{2 n}}{g^{2 n_{0}}}} \frac{\alpha^{n}}{\sqrt{n !}}
\end{aligned}
$$

with probabilities

$$
\begin{aligned}
& p_{\mathrm{s}}=\exp \left(-r^{2}\right)\left(\sum_{n=0}^{n_{0}} \frac{g^{n} r^{2 n}}{n ! g^{n_{0}}}+\sum_{n=n_{0}+1}^{\infty} \frac{r^{2 n}}{n !}\right) \\
& p_{\mathrm{f}}=\exp \left(-r^{2}\right) \sum_{n=0}^{n_{0}}\left(1-\frac{g^{2 n}}{g^{2 n_{0}}}\right) \frac{r^{2 n}}{n !}
\end{aligned}
$$




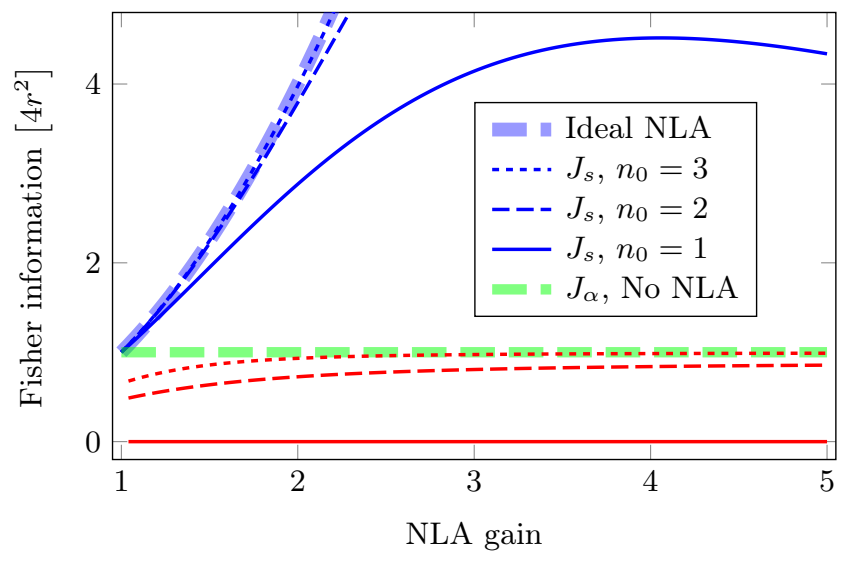

FIG. 3. Fisher information of successful and failed amplification events versus NLA gain. $J_{\mathrm{s}}$ (blue lines) is the Fisher information when the NLA successfully amplify the state. In these case, the Fisher information is higher than the Fisher information without the NLA, $J_{\alpha}$ (green line). $J_{\mathrm{f}}$ (red lines) is the Fisher information when the NLA failed to amplify the state. For these case, $J_{\mathrm{f}}$ is lesser than $J_{\alpha}$. $J_{\text {ideal }}$ (thick blue line) is the Fisher information of the state $|g \alpha\rangle$ that one will obtain from a successful NLA with a large $n_{0}$. Input state has amplitude $r=0.25$ and the Fisher information are normalised such that $J_{\alpha}=1$.

that do not depend on $\theta$. The probability of success and failure are plotted in Fig. 2 for $r=0.25$. As $n_{0}$ increases, we get a better approximation to the ideal NLA transformation but at the expense of a lower probability of success. Differentiating the outputs, we get the unnormalised states

$\left|\dot{\psi}_{\mathrm{s}}\right\rangle=\frac{\exp \left(\frac{-r^{2}}{2}\right)}{\sqrt{p_{\mathrm{s}}(r)}}\left(\sum_{n=0}^{n_{0}}|n\rangle \frac{i(g \alpha)^{n} n}{\sqrt{n !} g^{n_{0}}}+\sum_{n=n_{0}+1}^{\infty}|n\rangle \frac{i \alpha^{n} n}{\sqrt{n !}}\right)$

$\left|\dot{\psi}_{\mathrm{f}}\right\rangle=\frac{\exp \left(\frac{-r^{2}}{2}\right)}{\sqrt{p_{\mathrm{f}}(r)}} \sum_{n=0}^{n_{0}}|n\rangle \sqrt{1-\frac{g^{2 n}}{g^{2 n_{0}}}} \frac{i \alpha^{n} n}{\sqrt{n !}}$

with which we can compute $J_{\mathrm{s}}$ and $J_{\mathrm{f}}$.

We plot the Fisher information $J_{\alpha}, J_{\mathrm{s}}$ and $J_{\mathrm{f}}$ as a function of NLA gain in Fig. 3. The successfully amplified states $\left|\psi_{\mathrm{s}}\right\rangle$ have higher Fisher information compared to the input coherent states, while the failure states $\left|\psi_{\mathrm{f}}\right\rangle$ have a lower Fisher information. Hence, we can probabilistically get a higher information when the amplification succeed. For $n_{0}=1$, the states $\left|\psi_{\mathrm{f}}\right\rangle$ carries no information about the phase $\theta$. In Fig. 4. we plot the Fisher information scaled by their respective probabilities. We see that $p_{\mathrm{s}} J_{\mathrm{s}}$ and $p_{\mathrm{f}} J_{\mathrm{f}}$ are both lower than $J_{\alpha}$. Their sum $J_{\mathrm{NLA}}$, is also always lower than the Fisher information without using an NLA. This demonstrates the fact that doing a post-selection cannot increase information 11, 13, 14. From Fig. 2, we see that when $g$ increases, there is a much higher probability for the amplification to fail. For $n_{0}>1$, this results in more net

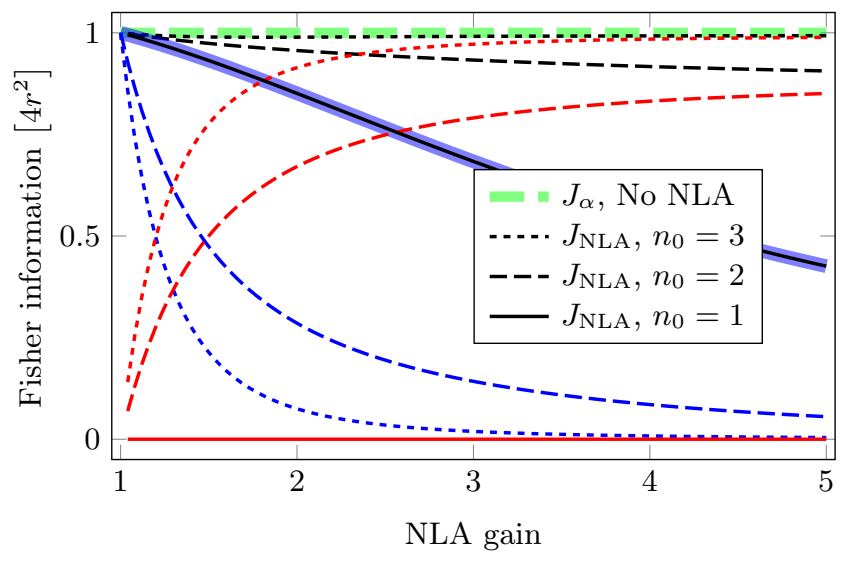

FIG. 4. Breakdown of the Fisher informations of the NLA when $p_{\mathbf{s}}=n_{\mathbf{s}} / m$ versus NLA gain. The scaled information from the successful NLA events $p_{\mathrm{s}} J_{\mathrm{s}}$ (blue lines) decreases as the NLA gains increases due to the low probability of success, while scaled information from the failed NLA events $p_{\mathrm{f}} J_{\mathrm{f}}$ (red lines) increases with higher gain. Their sum gives the net information $J_{\mathrm{NLA}}$ (black lines) which is always lower than the Fisher information one gets without the NLA $J_{\alpha}$ (green line). Input state has amplitude $r=0.25$ and the Fisher information are normalised such that $J_{\alpha}=1$.

information gained from the failed amplification events than the successfully amplified events at high $g$.

In Fig. 5, we fix the NLA gain $g=2$, and plot the Fisher information $J_{\mathrm{NLA}}$ as a function of the fraction of successfully amplified states $n_{\mathrm{s}} / m$. We see that as $n_{\mathrm{s}}$ increases, $J_{\mathrm{NLA}}$ increases and eventually becomes larger than $J_{\alpha}$. However, the probability to get a large enough $n_{\mathrm{s}}$ is small when the sample size $m$ is large. For example, for $m=1000$, we need $n_{\mathrm{s}}>89$ before $J_{\mathrm{NLA}}>J_{\alpha}$. The probability for this is only $4.68 \%$. The vertical line indicates the mean value of $n_{\mathrm{s}} / m=p_{\mathrm{s}}$. At this value, $J_{\mathrm{NLA}}$ is less than $J_{\alpha}$.

\section{SIMULATIONS WITH FINITE SAMPLE}

For small $\theta$ and pure state $\rho_{\theta}$, the QCR bound can be attained by measuring the observable $\mathcal{C}=\lambda^{2} \mathcal{L}$ where $\mathcal{L}=2\left(\left|\psi_{0}\right\rangle\left\langle\dot{\psi}_{0}|+| \dot{\psi}_{0}\right\rangle\left\langle\psi_{0}\right|\right)$ has rank at most two and $\lambda^{2}=1 /\left(4 \operatorname{Tr}\left[\rho_{0} \dot{\rho}_{0}^{2}\right]\right)$. The estimator obtained through $\mathcal{C}$ has moments

$$
\begin{aligned}
\operatorname{Tr}\left[\rho_{\theta} \mathcal{C}\right] & =\theta+O\left(\theta^{2}\right) \\
\operatorname{Tr}\left[\rho_{\theta} \mathcal{C}^{2}\right] & =\lambda^{2}+O\left(\theta^{2}\right)
\end{aligned}
$$

which verify that $\mathcal{C}$ is an unbiased estimator of $\theta$ achieving the $\mathrm{QCR}$ bound. The observable $\mathcal{C}$ has zero trace and spectral decomposition $\left|c_{+}\right\rangle \lambda\left\langle c_{+}|-| c_{-}\right\rangle \lambda\left\langle c_{-}\right|$where $\left|c_{+}\right\rangle$and $\left|c_{-}\right\rangle$are orthonormal vectors. Given $m$ trials, the probability of obtaining $n_{+}$positive outcomes and $n_{-}$negative outcomes follows a multinomial distribution $\operatorname{Pr}\left(n_{+}, n_{-}\right)=\frac{m}{n_{+} ! n_{-} ! n_{0} !} p_{+}^{n_{+}} p_{-}^{n_{-}} p_{0}^{n_{0}}$ where $n_{0}=$ 


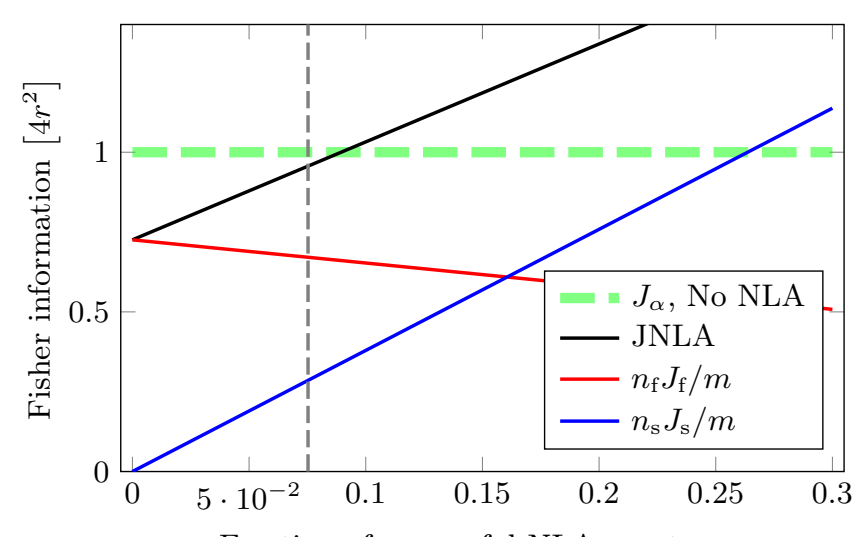

Fraction of successful NLA events, $n_{s}$

FIG. 5. Breakdown of the Fisher informations of the NLA versus fraction of successfully amplified states $n_{\mathbf{s}} / m$. Fisher information from the successfully amplified states (blue line) increases when the fraction of successful NLA events increases while the Fisher information from the unsuccessfully amplified states (red line) decreases. The net Fisher information (black line) can be higher than the Fisher information one gets without using the NLA $J_{\alpha}$ (green line) when $n_{\mathrm{s}}$ is large enough. The probability of this happening is small when the sample size $m$ is large. The vertical line denotes the most likely $n_{\mathrm{s}}=m p_{\mathrm{s}}$. On this line, $J_{\mathrm{NLA}}$ is less than $J_{\alpha}$. Input state has amplitude $r=0.25$. The NLA has a gain $g=2$ and $n_{0}=2$. The Fisher information are normalised such that $J_{\alpha}=1$.

$m-n_{-}-n_{+}$and the event probabilities

$$
\begin{aligned}
p_{ \pm} & =\left\langle c_{ \pm}\left|\rho_{\theta}\right| c_{ \pm}\right\rangle \\
& =\frac{1}{2}\left(1 \pm \frac{\theta}{\lambda}\right)+O\left(\theta^{2}\right)
\end{aligned}
$$

and $p_{0}=1-p_{-}-p_{+}$.

For coherent states without the NLA, $\lambda_{\alpha}=1 /(2 r)$ and $\mathcal{C}_{\alpha}=\mathcal{L}_{\alpha} /\left(4 r^{2}\right)$ with

$$
\begin{aligned}
\operatorname{Tr}\left[\mathcal{C}_{\alpha} \rho_{\alpha}\right] & =\theta+O\left(\theta^{2}\right) \\
\operatorname{Tr}\left[\mathcal{C}_{\alpha}^{2} \rho_{\alpha}\right] & =\frac{1}{4 r^{2}}+O\left(\theta^{2}\right)
\end{aligned}
$$

is an optimal unbiased estimator of $\theta$. For $m$ measurements, the counts $n_{\alpha+}$ and $n_{\alpha-}$ follows a multinomial distribution with $m$ trials and event probabilities $p_{\alpha \pm}=\left\langle c_{\alpha \pm}\left|\rho_{\theta}\right| c_{\alpha \pm}\right\rangle . \mathcal{C}_{\alpha}$ is the maximum likelihood estimator giving an estimate [27]

$$
\hat{\theta}_{\alpha}=\frac{n_{\alpha+}-n_{\alpha-}}{n_{\alpha+}+n_{\alpha-}} \lambda_{\alpha}
$$

The estimate obtained from the NLA can be viewed as an estimate obtained from a five outcome POVM $\left\{E_{\mathrm{s} \pm}^{2}, E_{\mathrm{f} \pm}^{2}, E_{0}^{2}\right\}$, where $E_{\mathrm{s} \pm}^{2}=E_{\mathrm{s}}\left|c_{\mathrm{s} \pm}\right\rangle\left\langle c_{\mathrm{s} \pm}\right| E_{\mathrm{s}}, E_{\mathrm{f} \pm}^{2}=$ $E_{\mathrm{f}}\left|c_{\mathrm{f} \pm}\right\rangle\left\langle c_{\mathrm{f} \pm}\right| E_{\mathrm{f}}$ and $E_{0}^{2}=1-E_{\mathrm{s}+}^{2}-E_{\mathrm{s}-}^{2}-E_{\mathrm{f}+}^{2}-E_{\mathrm{f}-}^{2}$. The vectors $\left|c_{\mathrm{s}}\right\rangle$ and $\left|c_{\mathrm{f} \pm}\right\rangle$ are the eigenvectors of the observable $\mathcal{C}_{\mathrm{s}}$ and $\mathcal{C}_{\mathrm{f}}$ with corresponding eigenvalues $\lambda_{\mathrm{s}}$ and $\lambda_{\mathrm{f}}$ for optimal estimation with the input states $\left|\psi_{\mathrm{s}}\right\rangle$ and $\left|\psi_{\mathrm{f}}\right\rangle$.

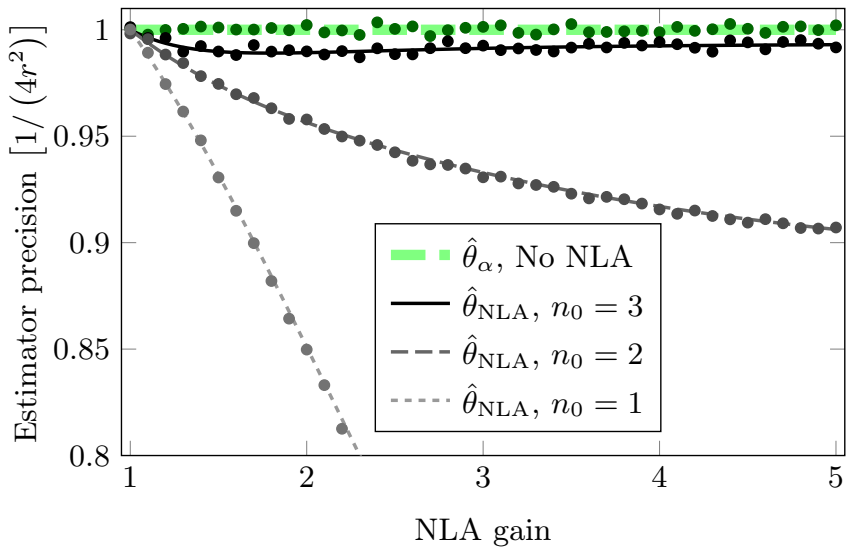

FIG. 6. Simulation of estimator precision for NLA versus NLA gain. The measurement precision using the NLA (black lines) is always worse than without using the NLA (green line). Increasing the NLA gain does not make it better. Estimates were obtained from a sample size of $m=1000$ input states with $r=0.25$ and $\theta_{\text {true }}=0.01$. The datapoints were obtained by measuring the precision from $10^{6}$ estimation runs. The lines are the asymptotic theoretical precisions conditioned on the most likely values of $n_{s}$ and $n_{f}$. The precision is scaled such that $\operatorname{Precision}\left(\hat{\theta}_{\alpha}\right)=1$.

Given $m$ measurements, the count rates $n_{\mathrm{s} \pm}$ and $n_{\mathrm{f} \pm}$ follows a multinomial distribution with $m$ trials and event probabilities $p_{\mathrm{s} \pm}=\operatorname{Tr}\left[\rho_{\alpha} E_{\mathrm{s} \pm}^{2}\right]$ and $p_{\mathrm{f} \pm}=\operatorname{Tr}\left[\rho_{\alpha} E_{\mathrm{f} \pm}^{2}\right]$. Given these counts, the maximum likelihood estimate for $\theta$ is constructed by 27

$$
\hat{\theta}_{\mathrm{NLA}}=\frac{n_{\mathrm{s}} \lambda_{\mathrm{f}}^{2}}{n_{\mathrm{s}} \lambda_{\mathrm{f}}^{2}+n_{\mathrm{f}} \lambda_{\mathrm{s}}^{2}} \hat{\theta}_{\mathrm{s}}+\frac{n_{\mathrm{f}} \lambda_{\mathrm{s}}^{2}}{n_{\mathrm{s}} \lambda_{\mathrm{f}}^{2}+n_{\mathrm{f}} \lambda_{\mathrm{s}}^{2}} \hat{\theta}_{\mathrm{f}},
$$

which is consistent with Eq. (5) and where $n_{\mathrm{s}}=n_{\mathrm{s}+}+n_{\mathrm{s}-}$ and $n_{\mathrm{f}}=n_{\mathrm{f}+}+n_{\mathrm{f}-}$. The intermediate estimators are $\hat{\theta}_{\mathrm{s}}=\left(n_{\mathrm{s}+}-n_{\mathrm{s}-}\right) \lambda_{\mathrm{s}} / n_{\mathrm{s}}$ and $\hat{\theta}_{\mathrm{f}}=\left(n_{\mathrm{f}+}-n_{\mathrm{f}-}\right) \lambda_{\mathrm{f}} / n_{\mathrm{f}}$. We plot the precision of the estimators $\hat{\theta}_{\alpha}$ and $\hat{\theta}_{\mathrm{NLA}}$ defined by

$$
\operatorname{Precision}(\hat{\theta})=\frac{1}{m \operatorname{MSE}(\hat{\theta})}
$$

in Fig. 6, where the mean square error (MSE) of an estimator $\hat{\theta}$ is

$$
\operatorname{MSE}(\hat{\theta})=\mathbb{E}_{\hat{\theta}}\left[\left(\hat{\theta}-\theta_{\text {true }}\right)^{2}\right] \geq \operatorname{Var}(\hat{\theta}) .
$$

Here $\theta_{\text {true }}$ is the true value of the parameter $\theta$. From Fig. 6, we see that on average, the NLA does not increase the precision for phase estimation.

\section{DISCUSSION}

The NLA is well suited for some tasks where all that matters are the successfully amplified states and when 
the probability of success does not matter, such as in probabilistic entanglement distillation and quantum key distribution [7]. In a phase estimation problem, if the figure of merit is the precision from a given number of sample, then as to be expected, using the NLA does not offer any advantage for phase estimation when compared to the optimal phase estimation scheme.

However with different figure of merits, using an NLA and post-selecting only successfully amplified events can help in metrology. Suppose we associate a cost $x$ for acquiring a sample, $y$ for direct measurement of an estimator observable from each sample and $z$ for applying a noiseless linear amplification on a sample, and our objective is to minimize the cost for obtaining an estimate for $\theta$ to a specified precision $\epsilon$. In order to achieve the specified precision without using the NLA, we would need to perform an estimate on $m_{\alpha}=\epsilon / J_{\alpha}$ samples. The total cost is then $\epsilon(x+y) / J_{\alpha}$. With the NLA, and performing an estimate only when the NLA heralds a successful amplification event, we now need to perform an estimate on only $m_{\mathrm{s}}=\epsilon / J_{\mathrm{s}}$ samples. Since $J_{\mathrm{s}}>J_{\alpha}$, each measurement gives more information and so we need less estimator measurements compared to estimating without the NLA. However the total number of samples we need to acquire increases because some samples were discarded when the NLA did not herald a successful amplification. We now need on average a total of $m_{\mathrm{s}} / p_{\mathrm{s}}$ samples and the total cost of the estimate would be $\epsilon\left(x+z+p_{\mathrm{s}} y\right) /\left(p_{\mathrm{s}} J_{\mathrm{s}}\right)$. In conventional metrology, the cost $y$ assigned to measuring an estimator observable is zero, and since $p_{\mathrm{s}} J_{\mathrm{s}}<J_{\alpha}$, the cost from the post-selection strategy will always be higher than without using the NLA. In this case, post-selection does not help. However if $y$ is non-zero, then the total cost of using the NLA and performing post-selection can be lesser than a direct measurement on all samples. This is true when

$$
y>\frac{\left(J_{\alpha}-p_{\mathrm{s}} J_{\mathrm{s}}\right) x+J_{\alpha} z}{p_{\mathrm{s}}\left(J_{\mathrm{s}}-J_{\alpha}\right)} .
$$

In this case, the better strategy would be to abstain from measuring the sample whenever the NLA fails to amplify.

Acknowledgements This research is supported by the Australian Research Council (ARC) under the Centre of Excellence for Quantum Computation and Communication Technology (CE110001027)
[1] T. C. Ralph and A. P. Lund. Nondeterministic noiseless linear amplification of quantum systems. AIP Conference Proceedings, 1110(1):155-160, 2009.

[2] Carlton Caves. Quantum limits on noise in linear amplifiers. Phys. Rev. D, 26(8):1817-1839, Oct 1982.

[3] Mario A. Usuga, Christian R. Mller, Christoffer Wittmann, Petr Marek, Radim Filip, Christoph Marquardt, Gerd Leuchs, and Ulrik L. Andersen. Noisepowered probabilistic concentration of phase information. Nat Phys, 6(10):767-771, Oct 2010.

[4] G. Y. Xiang, T. C. Ralph, A. P. Lund, N. Walk, and G. J. Pryde. Heralded noiseless linear amplification and distillation of entanglement. Nature Photonics, 4(5):316319, Mar 2010.

[5] Franck Ferreyrol, Marco Barbieri, Rémi Blandino, Simon Fossier, Rosa Tualle-Brouri, and Philippe Grangier. Implementation of a nondeterministic optical noiseless amplifier. Phys. Rev. Lett., 104(12):123603, 2010.

[6] Franck Ferreyrol, Rémi Blandino, Marco Barbieri, Rosa Tualle-Brouri, and Philippe Grangier. Experimental realization of a nondeterministic optical noiseless amplifier. Phys. Rev. A, 83(6):063801, 2011.

[7] Helen M. Chrzanowski, Nathan Walk, Syed M. Assad, Jiri Janousek, Sara Hosseini, Timothy C. Ralph, Thomas Symul, and Ping Koy Lam. Measurement-based noiseless linear amplification for quantum communication. Nature Photonics, 8(4):333-338, Mar 2014.

[8] J. Fiurasek. Optimal probabilistic estimation of quantum states. New J. Phys., 8(9):192192, Sep 2006.

[9] Bernat Gendra, Elio Ronco-Bonvehi, John Calsamiglia, Ramon Muñoz-Tapia, and Emilio Bagan. Beating noise with abstention in state estimation. New Journal of Physics, 14(10):105015, Oct 2012.
[10] B. Gendra, E. Ronco-Bonvehi, J. Calsamiglia, R. MuozTapia, and E. Bagan. Quantum metrology assisted by abstention. Physical Review Letters, 110(10), Mar 2013.

[11] Saki Tanaka and Naoki Yamamoto. Information amplification via postselection: A parameter-estimation perspective. Phys. Rev. A, 88:042116, Oct 2013.

[12] Christopher Ferrie and Joshua Combes. Weak value amplification is suboptimal for estimation and detection. Phys. Rev. Lett., 112(4), Jan 2014.

[13] Joshua Combes, Christopher Ferrie, Zhang Jiang, and Carlton M. Caves. Quantum limits on postselected, probabilistic quantum metrology. Phys. Rev. A, 89:052117, May 2014.

[14] Lijian Zhang, Animesh Datta, and Ian A. Walmsley. Precision metrology using weak measurements. Phys. Rev. Lett., 114(21), May 2015.

[15] Joshua Combes and Christopher Ferrie. Cost of postselection in decision theory. Phys. Rev. A, 92(2), Aug 2015.

[16] Carl W Helstrom. Quantum detection and estimation theory. Academic press, 1976.

[17] A Holevo. Probabilistic and Statistical Aspects of Quantum Theory. North-Holland, Amsterdam, 1982.

[18] Samuel L. Braunstein and Carlton M. Caves. Statistical distance and the geometry of quantum states. Phys. Rev. Lett., 72(22):3439-3443, May 1994.

[19] D. Petz and C. Ghinea. Introduction to Quantum Fisher Information, chapter 15, pages 261-281. World Scientific, Jan 2011.

[20] O E Barndorff-Nielsen and R D Gill. Fisher information in quantum statistics. Journal of Physics A: Mathematical and General, 33(24):44814490, Jun 2000.

[21] Akio Fujiwara and Hiroshi Nagaoka. Quantum fisher 
metric and estimation for pure state models. Phys. Lett. A, 201(2-3):119124, May 1995.

[22] Shashank Pandey, Zhang Jiang, Joshua Combes, and Carlton M. Caves. Quantum limits on probabilistic amplifiers. Phys. Rev. A, 88(3), Sep 2013.

[23] N. A. McMahon, A. P. Lund, and T. C. Ralph. Optimal architecture for a nondeterministic noiseless linear amplifier. Phys. Rev. A, 89:023846, Feb 2014.

[24] E. Bagan, A. Monras, and R. Muñoz-Tapia. Phase vari- ance of squeezed vacuum states. Phys. Rev. A, 78:043829, Oct 2008 .

[25] M. Aspachs, J. Calsamiglia, R. Muñoz-Tapia, and E. Bagan. Phase estimation for thermal Gaussian states. Phys. Rev. A, 79(3), Mar 2009.

[26] O. Pinel, P. Jian, N. Treps, C. Fabre, and D. Braun. Quantum parameter estimation using general singlemode gaussian states. Phys. Rev. A, 88:040102, Oct 2013.

[27] John Rice. Mathematical statistics and data analysis. Nelson Education, 2006. 